\title{
The FDA should test the safety of ECT machines*
}

\author{
Peter R. Breggin** \\ Director, Empathic Therapy Center, www.empathictherapy.org \\ 101 East State Street, No. 112, Ithaca, NY 14850, USA \\ E-mail: psychiatricdrugfacts@hotmail.com
}

\begin{abstract}
Electroconvulsive therapy (ECT) and the machines that deliver it have never been tested for safety and efficacy in order to receive approval from the FDA. The American Psychiatric Association and ECT advocates protested when the FDA took steps to classify the machines as posing "an unreasonable risk of illness or injury", which would have required their testing before approval. Without requiring this testing, the FDA is now preparing to classify the treatment and the machines as safe. This article reviews evidence demonstrating that ECT is very harmful to the brain and mind, and concludes that the FDA should demand the usual testing, starting with animals, that is required before psychiatric treatments and machines are approved for marketing and use.
\end{abstract}

\section{Introduction}

Since its inception in the late 1930s, electroconvulsive therapy (ECT) has never been subjected to testing for Food and Drug Administration (FDA) approval in regard to safety and effectiveness. In 1979 the FDA classified ECT devices as demonstrating "an unreasonable risk of illness or injury" [9]. This would have required animal testing and additional evidence for safety.

Under heavy criticism from organized psychiatry and ECT advocates, the FDA panel then recommended reclassifying the treatment as safe for depressed patients [3, 9]. However, the panel also recommended delaying the approval process until engineering safety standards could be established for the machines. No further evaluation took place and ECT remained unregulated by the FDA.

Once again, the FDA is considering reclassifying ECT as safe. The agency should not do this. The following overview of the safety and effectiveness of electroconvulsive therapy addresses the necessity for requiring ECT to be subjected to the regulatory process as posing an "unreasonable risk of illness or injury".

\footnotetext{
*This report was submitted to the FDA on December 5, 2009 as an attachment to a Notice regarding Medical Devices: Neurological Devices; Electroconvulsive Therapy Device; Establishing a Public Docket (FDA-2009-N-0392-0001). The present paper appears simultaneously in Ethical Human Psychology and Psychiatry, and is published with permission of Springer Publishing Company, NY, USA.

**Dr. Breggin is Founder and Director Emeritus, International Center for the Study of Psychiatry and Psychology.
} 


\section{Overview}

\subsection{There is a consensus within psychiatry, even among staunch advocates of electroconvulsive therapy (ECT), that ECT is the most controversial treatment in psychiatry}

The Consensus Development Conference on ECT [8] conducted by NIH stated, "Electroconvulsive therapy is the most controversial treatment in psychiatry" and referred to 45 years of disputes surrounding the treatment. In his introduction to Richard Abrams's [1] book in favor of ECT, Max Fink referred to "more than 50 years of controversy" surrounding the treatment. In addition, ECT is the most frequently regulated psychiatric treatment under state laws.

Given this extraordinary degree of controversy, there can be no justification for not subjecting ECT to the same scrutiny that is given to devices and treatments that are far less controversial, including at the least new animals studies. Adequately conducted large animal studies would once again show, as they have in the past (see below), that the treatment is too damaging to be tested in human trials. Only political pressure could account for not subjecting this treatment to a very thorough scrutiny, beginning with animal studies, before permitting its routine use in psychiatry.

\subsection{Because it causes an acute delirium, there can be no scientific doubt that ECT harms the brain and mental function. The only possible controversy surrounds the severity and persistence of the harm}

ECT produces sufficient trauma to the brain to cause a severe grand mal convulsion. All ECT treatments result in a period of coma lasting several minutes or more, sometimes including a flat line EEG. In routine application, the patient awakens in a delirium that is virtually indistinguishable from any other closed head injury. Typical symptoms include severe headache, memory dysfunction, disorientation, confusion, lack of judgment and unstable mood [1-3]. The treatment always results in apathy, and sometimes in euphoria, which are typical reactions to traumatic brain injury. Consent forms routinely warn patients not to make decisions during or shortly after the completion of any series of ECT treatments.

It is acknowledged in neurology that repeated head injuries that produce concussive symptoms are likely to cause persistent harm. ECT treatments are far more traumatic than most concussions, and include prolonged coma after each treatment, sometimes accompanied by EEG flat lining, and severe delirium after a few treatments or less. The number of traumatic ECT treatments usually far exceeds the number of concussions that produce lasting harm.

In the rational practice of regulatory affairs, the fact that a treatment causes such initial trauma would in itself require it to be withdrawn from the market; it certainly requires a thorough examination of ECT by the FDA, starting with animal studies.

\subsection{Multiple large animal studies have demonstrated generalized brain damage from ECT in the form of cell damage and death, and small pinpoint hemorrhages}

Animal ECT studies have often been conducted using clinical apparatus, relatively short treatment courses, and less electrical energy than currently used in clinical practice. The extensive animal research and the mechanisms of damage associated with ECT have been previously reviewed [2, 4, 5, 7]. Hartelius's (1955) book length monograph [10] on the impact of ECT on cats was the last experimental study published on large animals. The results were consistent with earlier studies on monkeys and dogs that demonstrated widespread pinpoint hemorrhages and neuronal damage and death. The Hartelius study was 
the last one conducted on large animals, probably because its results were so conclusive that researchers stopped trying to prove that ECT is safe when given to large animals. ECT damages the brains of animals, even when given in doses less traumatic than those currently used in routine clinical treatment.

There is no reason to believe that modified ECT using paralyzing agents and artificial respiration would be less damaging to the brain. The modifications were developed to prevent fractures, not to prevent harm to the brain. Instead, modified ECT adds the risk of anesthesia and requires higher doses of electricity to overcome the anti-convulsive effects of the anesthesia $[2,5]$. One cannot assume that modified ECT is safer; instead, the modified treatment must also be subjected to experimental study with large animals.

\subsection{Substantial new evidence confirms that ECT produces lasting memory dysfunction as well as more generalized persistent cognitive deficits}

The evidence for lasting memory dysfunction and other cognitive deficits following ECT has previously been reviewed $[2,5,6]$. However, there is less need to review this material at this time, because a recent (2007) controlled follow up of patients treated with ECT by Sackeim et al. [14] has confirmed generalized, lasting mental dysfunction (losses in "global cognitive status") consistent with a dementia, including a variety of deficits in memory and overall cognitive function $(p<0.0001)$. This finding is consistent with the known initial clinical impact of ECT, the animal literature, and clinical knowledge about the likelihood of persistent brain dysfunction following repeated traumatic head injury. The study involved 347 patients treated with the full range of currently available ECT techniques and evaluated with neuropsychological testing for up to six months. Except in regard to a psychiatric treatment, substantial evidence on this scale for persistent damage would lead to an inquiry into withdrawing a treatment from the market.

\subsection{ECT is especially devastating to the vulnerable brain and body of the elderly, a group in which ECT is very frequently used}

The impact of ECT on the elderly has previously been reviewed [2] (pp. 230-233). As would be predicted based on the fragility of the older brain, ECT causes more severe brain dysfunction in the elderly. More strikingly, ECT dramatically increases the mortality rate in the elderly. Kroessler and Fogel [11] followed up 65 patients 80 years old or older who were hospitalized for depression. Survival for the ECT group after 1, 2, and 3 years was only $73 \%, 54 \%$, and $51 \%$ respectively. Survival was much better for the drug-treated non-ECT group at 1, 2, and 3 years where it was $96 \%, 90 \%$ and $75 \%$.

\subsection{Despite several decades of effort, advocates for ECT have been unable to demonstrate any sustained improvement from ECT}

The Consensus Development Conference on ECT [8] found that controlled clinical trials failed to demonstrate any positive effect beyond four weeks. Thus the risk/benefit ratio is very poor. This fourweek period corresponds to the period of the acute delirium, when the ECT effects of emotional blunting and/or euphoria are mistaken for clinical improvement. Typically, the patient stops voicing complaints and may display an artificially elevated mood [2].

Repeated attempts to show a reduction in suicide risk have failed, and indeed the evidence is great for an increased suicide rate following ECT [2]. Most recently, Munk-Olsen et al. [12] found an overall increased rate of suicide in patients given ECT, including patients treated within the prior week (p. 437).

A variety of reviews have shown that sham ECT produces as much improvement as ECT itself, without any of the risks [13]. This is consistent with the good response to placebo found in most studies 
of depression. However, the question of efficacy is irrelevant given the frequent and potentially severe damage caused by ECT. The known risk of causing significant persistent brain dysfunction in a significant percentage of patients makes it too dangerous to use, even if it helps a significant percentage of individuals - which it does not. As already noted, the FDA should not test ECT on humans until large animals studies for safety have been completed.

\section{Summary and conclusions}

Given the already available information about the damage associated with ECT, medical ethics and sound medical science require it to be withdrawn from the market. In almost any other case, a treatment that caused such grossly obvious initial trauma to the brain and to mental function would not be permitted to remain on the market, regardless of whether or not it helped a substantial number of people. In the case of ECT, the most optimistic assessments of its efficacy indicate that the beneficial effect is limited to a period of about four weeks.

Even if it were not already known that ECT produces persistent brain dysfunction, and even if it were not already known that longer-term it produces no measurably positive effect, its clinically recognizable acute traumatic impact on the brain would require ECT to be withdrawn from the market pending further study. To allow it to persist in the marketplace without further inquiry into its safety would be unconscionable, regardless of any claims for its efficacy. In fact, the treatment is too damaging to be tested on human beings, and the FDA should begin by requiring renewed testing on large animals (Phase I studies), before deciding that it is safe to test on human beings. The FDA should not reclassify ECT as safe.

\section{References}

[1] R. Abrams, Electroconvulsive Therapy, Oxford University Press, New York, 1988.

[2] P. Breggin, Brain-Disabling Treatments in Psychiatry: Drugs, Electroshock, and the Psychopharmaceutical Complex, Second Edition, Chapter 9, "Electroshock for Depression", Springer Publishing Company, New York, 2008, pp. $217-251$.

[3] P. Breggin, Brain-Disabling Treatments in Psychiatry: Drugs, Electroshock, and the Role of the FDA. Chapter 8, "Electroshock for Depression”, Springer Publishing Company, New York, 1997, pp. 129-156.

[4] P. Breggin, Disabling the Brain with Electroshock, in: M. Dongier and D. Wittkower, eds, Divergent Views in Psychiatry, Harper \& Row, Hagerstown, Maryland.

[5] P. Breggin, Electroshock: Its Brain-Disabling Effects, Springer Publishing Company, New York, 1979.

[6] P. Breggin, Electroshock therapy and brain damage: The acute organic brain syndrome as treatment, Behavior and Brain Sciences 7 (1984), 24-25.

[7] P. Breggin, Neuropathology and cognitive dysfunction from ECT, Presented at the Consensus Development Conference on Electroconvulsive Therapy, sponsored by NIMH and NIH, Psychopharmacology Bulletin 22 (1986), 476-479.

[8] Consensus Conference on ECT, Journal of the American Psychiatric Association 245 (1985), 2103-2108.

[9] Food and Drug Administration (FDA), Neurological devices; proposed rule to reclassify the electroconvulsive device intended for use in treating severe depression. 21 CFR Part 882 [Docket No. 82P-0316] Federal Register 55(172), (1990, September 5), 36578-36590.

[10] H. Hartelius, Cerebral changes following electrically induced convulsions, Acta Psychiatrica Neurologica Scandinavica, 77(suppl.) (1952), 1-128.

[11] D. Kroessler and B. Fogel, Electroconvulsive therapy for major depression in the oldest old, American Journal of Geriatric Psychiatry 1 (1993), 30-37.

[12] T. Munk-Olsen, T. Laursen, P. Videbech, P. Mortensen and R. Rosenberg, All-cause mortality among recipients of electroconvulsive therapy. British Journal of Psychiatry 190 (2007), 435-439.

[13] C. Ross, The sham ECT literature: Implications for consent, Ethical Human Psychology and Psychiatry 8 (2006), 17-28.

[14] J. Sackeim, J. Prudic, R. Fuller, J. Keilp, P. Lavori and M. Olson, The cognitive effects of electroconvulsive therapy in community settings, Neuropsychopharmacology 32 (2007), 244-254. 